\title{
Evidence of a Christmas spirit network in the brain: functional MRI study
}

\author{
(®) $(1) \Theta$ OPEN ACCESS
}

\begin{abstract}
Anders Hougaard research fellow in neurology ${ }^{1}$, Ulrich Lindberg research fellow in neuroimaging ${ }^{2}$, Nanna Arngrim research fellow in neurology ${ }^{1}$, Henrik B W Larsson professor of clinical physiology ${ }^{2}$, Jes Olesen professor of neurology ${ }^{1}$, Faisal Mohammad Amin research fellow in neurology ${ }^{1}$, Messoud Ashina professor of neurology ${ }^{1}$, Bryan T Haddock medical physicist ${ }^{2}$
\end{abstract}

'Danish Headache Centre and Department of Neurology, Rigshospitalet, Glostrup, Faculty of Health and Medical Sciences, University of Copenhagen, Denmark; ${ }^{2}$ Functional Imaging Unit and Department of Clinical Physiology, Nuclear Medicine and PET, Rigshopitalet, Glostrup, Faculty of Health and Medical Sciences, University of Copenhagen, Denmark

\begin{abstract}
Objective To detect and localise the Christmas spirit in the human brain.

Design Single blinded, cross cultural group study with functional magnetic resonance imaging ( $\mathrm{fMRI}$ ).

Setting Functional imaging unit and department of clinical physiology, nuclear medicine and PET in Denmark.

Participants 10 healthy people from the Copenhagen area who routinely celebrate Christmas and 10 healthy people living in the same area who have no Christmas traditions.

Main outcome measures Brain activation unique to the group with Christmas traditions during visual stimulation with images with a Christmas theme.

Methods Functional brain scans optimised for detection of the blood oxygen level dependent (BOLD) response were performed while participants viewed a series of images with Christmas themes interleaved with neutral images having similar characteristics but containing nothing that symbolises Christmas. After scanning, participants answered a questionnaire about their Christmas traditions and the associations they have with Christmas. Brain activation maps from scanning were analysed for Christmas related activation in the "Christmas" and "non-Christmas" groups individually. Subsequently, differences between the two groups were calculated to determine Christmas specific brain activation.

Results Significant clusters of increased BOLD activation in the sensory motor cortex, the premotor and primary motor cortex, and the parietal lobule (inferior and superior) were found in scans of people who celebrate Christmas with positive associations compared with scans in a group having no Christmas traditions and neutral associations. These cerebral areas have been associated with spirituality, somatic senses, and recognition of facial emotion among many other functions.
\end{abstract}

Conclusions There is a "Christmas spirit network" in the human brain comprising several cortical areas. This network had a significantly higher activation in a people who celebrate Christmas with positive associations as opposed to a people who have no Christmas traditions and neutral associations. Further research is necessary to understand this and other potential holiday circuits in the brain. Although merry and intriguing, these findings should be interpreted with caution.

\section{Introduction}

The Christmas spirit has been a widespread phenomenon for centuries, commonly described as feelings of joy and nostalgia mixed with associations to merriment, gifts, delightful smells, and copious amounts of good food. It is yet to be determined, however, where in the human body this "Christmas spirit" resides and which biological mechanisms are involved. We attempted to localise the Christmas spirit in the human brain using functional magnetic resonance imaging (fMRI).

Since its inception in the early nineties, fMRI has been instrumental in neuropsychological studies localising emotional and functional centres in the human brain. Feelings such as joy, sorrow, and disgust have been isolated to certain cerebral regions. ${ }^{1}$ We used a similar technique by comparing a group of people who have celebrated Christmas since their youth with a group having no Christmas traditions. We scanned the two groups while they were viewing various images and analysed changes in brain activity when they were viewing images with yuletide themes as opposed to regular images. Our hypothesis was that the two groups would respond differently to Christmas images based on their differences in exposure to Christmas celebrations. 
Throughout the world, we estimate that millions of people are prone to displaying Christmas spirit deficiencies after many years of celebrating Christmas. We refer to this as the "bah humbug" syndrome. Accurate localisation of the Christmas spirit is a paramount first step in being able to help this group of patients. Location of the Christmas spirit could also contribute to a more general understanding of the brain's role in festive cultural traditions, making a medical contribution to cross cultural festivities and goodwill to all.

\section{Methods \\ Participants}

The fMRI data in this study were collected as part of the visual paradigm for healthy controls in a previously published migraine study. ${ }^{2}$ The study was undertaken in accordance with the Helsinki Declaration as revised in 2008 and was approved by the local ethics committee. All participants gave written and verbal consent to fMRI scanning during visual stimulation. A total of 26 participants were asked to fill out a questionnaire about their Christmas traditions, feelings associated with Christmas, and ethnicity after scanning based on an assumption of their cultural background (box). Participants, and the ethics committee, gave explicit consent to the use of control fMRI data from the study in this article. No eggnog or gingerbread was consumed before the scans.

\section{Experimental setup}

Participants were scanned with MRI while they were watching a series of images through video goggles (NordicNeuroLab, Bergen, Norway). A continual series of 84 images were displayed for two seconds each and were organised such that after six consecutive images with a Christmas theme there were six everyday images with similar forms and features though devoid of anything symbolising Christmas (fig $1 \Downarrow$ ). The alternating sets of Christmas and everyday images gave an interleaved block stimulation with the time periods where Christmas images are being viewed as "stimulation blocks" interleaved with "resting blocks" of viewing everyday images. Participants were informed that different images would be presented and were not made aware of there being a Christmas theme in the study.

Acquisition of MRI data was carried out on a 3T Philips Achieva (Philips Medical, Best, Netherlands). A T $\mathrm{T}_{1}$-weighted MPRAGE sequence was acquired for use as anatomical reference (150 sagittal slices; $1 \times 1 \times 1.1 \mathrm{~mm}$; TR=6.9 ms; TE=2.78 ms; flip angle=9). Functional scans used an echo planar imaging sequence $(\mathrm{TR}=3 \mathrm{~s} ; \mathrm{TE}=35 \mathrm{~ms}$; flip angle $=90$; voxel size $=1.8 \times 1.8 \times 4 \mathrm{~mm} ; 112$ volumes). Cerebral perfusion was imaged with a multi-TI pulsed arterial spin labelling sequence. ${ }^{3}$ Seven slices centred at the glabella were acquired to investigate regional perfusion and for use as covariate in the functional general linear model.

We carried out all post-processing, including the creation of brain activation maps, with FSL tools (FMRIB, Oxford) as described by Jenkinson and colleagues, ${ }^{4}$ with default settings. With FSL, functional data were motion corrected, spatially filtered with a full width half maximum Gaussian kernel of 5 $\mathrm{mm}$, before we carried out general linear modelling (GLM) with the FMRI expert analysis tool (FEAT). Functional images were spatially normalised to the MNI-152 standard brain. We determined significant clusters of changes in brain activity (changes in BOLD signal) when participants were viewing Christmas images from the $\mathrm{z}$ statistical images by a threshold of $z>2.3$ and a (corrected) cluster significance threshold of
$\mathrm{P}<0.05$. We applied family-wise error correction (FWE). Group comparison based on two sample $t$ test was likewise performed with FEAT with default settings. Perfusion measurements were analysed with the QUASIL tool (part of FSL), where we calculated quantification according to Petersen and colleagues. ${ }^{3}$ Locations of activation clusters from viewing Christmas images were cross referenced with the Jüelich atlas of the brain in FSL. To evaluate the methods, we previously performed a pilot study in four participants (not included in the current experiment) using a similar design. The preliminary results of this study have been published in Danish. ${ }^{5}$

\section{Patient involvement}

No patients were involved in setting the research question or the outcome measures, nor were they involved in the design and implementation of the study. There are no plans to involve patients in dissemination.

\section{Results}

Based on the results of the questionnaire, 10 participants were allocated to the "Christmas group" (eight men, two women) and 10 to the "non-Christmas group" (eight men, two women). The six remaining participants were excluded either because of a strong Christmas connection despite having no tradition of celebrating Christmas ( $n=2)$ or non-positive associations with Christmas despite having a cultural background involving regular Christmas celebration. We analysed MRI data only from included participants. Those in the "Christmas group" were ethnic Danes who celebrated Christmas according to Danish tradition, while those in the "non-Christmas group" were Pakistani $(n=2)$, Indian $(n=2)$, Iraqi $(n=1)$, or Turkish $(n=2)$ expatriates or people of Pakistani descent $(n=3)$ who were born in Denmark.

The baseline perfusion scans showed a normal cerebral perfusion of $54 \mathrm{~mL} / 100 \mathrm{~g} / \mathrm{min}$ without any significant difference between the two groups $(\mathrm{P}=0.26)$. Activation maps from fMRI scans showed an increase of brain activity in the primary visual cortex $(\mathrm{P}<0.001)$ of both groups when the images viewed had a Christmas theme compared with the everyday images (fig $2 \Downarrow$ ). The Christmas group also had significant increases in neural activations in the primary somatosensory cortex when the images had a Christmas theme (fig $2 \Downarrow$ ). Comparison of the brain activation maps of the two groups showed five areas where the Christmas group responded to Christmas images with a higher activation than the non-Christmas group (fig $3 \Downarrow$ ). These areas of difference include the left primary motor and premotor cortex, right inferior/superior parietal lobule, and bilateral primary somatosensory cortex $(\mathrm{P}<0.001)$. In contrast, there were no areas of the brain where the non-Christmas group had significantly larger responses to Christmas images than the Christmas group.

\section{Discussion}

There is a cerebral response when people view Christmas images, and there are differences in this response between people who celebrate Christmas compared with those with no Christmas traditions. Cerebral perfusion was similar between the two groups, despite the Christmas group's yearly yuletide feast.

We identified a functional Christmas network comprising several cortical areas, including the parietal lobules, the premotor cortex, and the somatosensory cortex. Activation in these areas coincided well with our hypothesis that images with a Christmas theme would stimulate centres associated with the Christmas 


\section{Post-scan Christmas questionnaire}

Have you ever celebrated Christmas? (yes/no)

If yes, for how many years? (option of specifying number of years or answering "every year of my life")

If yes, what are your general feelings about Christmas? (generally positive/generally negative)

Have you lived in Denmark all of your life? (yes/no)

If no, for how long have you been living in Denmark? (option of specifying number of years)

If no, where have you been living before? (option of specifying countries)

What feeling do you associate with Christmas? (free text)

spirit. The left and right parietal lobules have been shown in earlier fMRI studies to play a determining role in self transcendence, the personality trait regarding predisposition to spirituality. ${ }^{6}$ Furthermore, the frontal premotor cortex is important for experiencing emotions shared with other individuals by mirroring or copying their body state, ${ }^{7}$ and premotor cortical mirror neurons even respond to observation of ingestive mouth actions. ${ }^{8}$ Recall of joyful emotions and pleasant ingestive behaviour shared with loved ones would be likely to elicit activation here. There is growing evidence that the somatosensory cortex plays an important role in recognition of facial emotion and retrieving social relevant information from faces. ${ }^{9}$ Collectively, these cortical areas possibly constitute the neuronal correlate of the Christmas spirit in the human brain.

We realise that some of our colleagues within the specialties of neuroscience and psychology, who we suspect could be afflicted by the aforementioned bah humbug syndrome, would argue that studies such as the present one overemphasise the importance of localised brain activity and that attempts to localise complex emotions in the brain contribute little to the understanding of these emotions. Citing a paper reporting fMRI evidence of brain activity in frozen salmon, ${ }^{10}$ representatives of this view have even coined terms for this practice such as "blobology," "neo-phrenology," "neuro-essentialism," and "neuro-bollocks" (Grinch and colleagues, personal communication). Naturally, in keeping with the good spirit of the holiday, we disagree with these negative perspectives.

We generally believe that fMRI is an outstanding technology for exploring the brain but that any fMRI experiment is only as good as its hypothesis, design, and interpretation. While celebrating the current results at a subsequent Christmas party, we discussed some limitations of the study. For instance, the study design doesn't distinguish whether the observed activation is Christmas specific or the result of any combination of joyful, festive, or nostalgic emotions in general. The paired Christmas/non-Christmas pictures might have been systematically different in a way that we were not aware of - for example, the "Christmas pictures" containing more red colour. Maybe the groups were different in other ways apart from the obvious cultural difference. Given these uncertainties and the risk of false positive results, our findings should ideally be reproduced before firm conclusions are drawn, especially when we consider the recently documented challenges of reproducibility in our neighbouring specialty of psychology. ${ }^{11}$ Bringing these issues up, however, really dampened the festive mood. Therefore we, in the best interest of the readers of course, decided not to ruin the good Christmas cheer for everyone by letting this influence our interpretation of the study.

Further research into this topic is necessary to identify the factors affecting one's response to Christmas. For example, responses to Christmas might change with development from a child, who primarily receives presents, to an adult, who primarily buys them. Subgroups subjected to receipt of tacky jumpers as their Christmas present might also have different responses in brain activity from those of subgroups who tend to receive more attractive gifts. Understanding how the Christmas spirit works as a neurological network could provide insight into an interesting area of human neuropsychology and be a powerful tool in treating ailments such as bah humbug syndrome. Comparative studies of these patterns will also be imperative in studying other seasonal disturbances, related to, for example, Easter, Chanukah, or Diwali. This study could therefore be an important first step in transcultural neuroscience and the associations humans have with their festive traditions.

We acknowledge all those who spread a spirit of warmth, kindness, and generosity, regardless of the season.

Contributors: AH (guarantor) and FMA developed the hypothesis, recruited and scanned the participants, and edited the paper. UL scanned the participants, performed the data analysis, created the figures, and edited the paper. NA scanned the participants and edited the paper. BTH assisted in the data analysis, interpreted the results, drafted and edited the paper, and is guarantor. HBWL provided the equipment, interpreted the results, and edited the paper. JO designed the study, interpreted the results, and edited the paper. MA coordinated and supervised the project, interpreted the results, and edited the paper. Funding: This research received no specific grant from any funding agency in the public, commercial, or not-for-profit sectors.

Competing Interests: All authors have completed the ICMJE uniform disclosure form and declare: no support from any organisation for the submitted work; no financial relationships with any organisations that might have an interest in the submitted work in the previous three years; no other relationships or activities that could appear to have influenced the submitted work. However, we would like to call "dibs" on any profitable non-invasive or even invasive treatment of bah humbug syndrome. We are currently preparing a patent application on a Santa's hat that you can buy for family members with symptoms. When they start grumbling at Christmas dinner, with the touch of a button you can give them electric stimulation right in the Christmas spirit centres.

Ethical approval: The ethics committee of the County of Copenhagen (H-KA-20060083) approved the study. All participants gave written informed consent to take part in the study.

Transparency: The lead author, $\mathrm{AH}$, affirms that this manuscript is an honest, accurate, and transparent account of the study being reported; that no important aspects of the study have been omitted; and that any discrepancies from the study as planned (and, if relevant, registered) have been explained.

Data sharing: All data are available by request from the corresponding author.

1 Lane RD, Reiman EM, Ahern GL, et al . Neuroanatomical correlates of happiness, sadness, and disgust. Am J Psychiatry 1997;154: 926-33. doi:10.1176/ajp.154.7. 926. 9210742

2 Hougaard A, Amin FM, Magon S, et al. No abnormalities of intrinsic brain connectivity in the interictal phase of migraine with aura. Eur J Neurol 2015;22: 702-e46. doi:10.1111/ ene.12636. 25573335

3 Pedersen ET. Brain perfusion imaging: quantification of cerebral blood flow using ASL Techniques. Aarhus Universitet, 2009.

4 Jenkinson M, Beckmann CF, Behrens TEJ, et al . FSL. Neuroimage 2012;62: 782-90. doi:10.1016/j.neuroimage.2011.09.015. 21979382 


\section{What is already known on this topic}

The Christmas spirit has eluded science thus far; though well known as a pleasant feeling, its cerebral location and mechanisms are still a mystery

Functional MRI has proved a valuable tool in locating which centres of the brain are active under a given stimulation such as viewing images

This technique has shown on several occasions that complex responses to stimulation evoke a network of activated areas in the brain

\section{What this study adds}

This study locates a "Christmas spirit" network in the brain that is activated by images with Christmas themes

The network showed a series of cerebral regions that are more active in people who celebrate Christmas with positive associations compared with people with no Christmas traditions and neutral associations

5. Amin FM, Lindberg U, Raghava JM, et al. The cerebral representation of the Christmas spirit. A transcultural functional magnetic resonance study. Ugeskr Laeger 2012;174: 3082-4. 23286725

6 Urgesi C, Aglioti SM, Skrap M, et al . The spiritual brain: selective cortical lesions modulate human self-transcendence. Neuron 2010;65: 309-19. doi:10.1016/j.neuron.2010.01. 026. 20159445

7 Balconi M, Bortolotti A. The "simulation" of the facial expression of emotions in case of short and long stimulus duration. The effect of pre-motor cortex inhibition by rTMS. Brain Cogn 2013;83: 114-20. doi:10.1016/j.bandc.2013.07.003. 23954669

8 Ferrari PF, Gallese V, Rizzolatti G, et al . Mirror neurons responding to the observation of ingestive and communicative mouth actions in the monkey ventral premotor cortex. Eur J Neurosci 2003:17: 1703-14 doi-10.1046/j.1460-9568.2003.02601 x. 12752388

9 Adolphs R, Damasio H, Tranel D, et al . A role for somatosensory cortices in the visual recognition of emotion as revealed by three-dimensional lesion mapping. $J$ Neurosci 2000;20: 2683-90. 10729349
10 Bennett CM, Baird AA, Miller MB. Neural correlates of interspecies perspective taking in the post-mortem atlantic salmon: an argument for proper multiple comparisons correction. Journal of Serendipitous and Unexpected Results 2011;1: 1-5.

11 Open Science Collaboration. Estimating the reproducibility of psychological science.

Science 2015; 349:aac4715. doi:10.1126/science.aac4716.

\section{Accepted: 21102015}

Published by the BMJ Publishing Group Limited. For permission to use (where not already granted under a licence) please go to http://group.bmj.com/group/rights-licensing/ permissions

This is an Open Access article distributed in accordance with the Creative Commons Attribution Non Commercial (CC BY-NC 3.0) license, which permits others to distribute, remix, adapt, build upon this work non-commercially, and license their derivative works on different terms, provided the original work is properly cited and the use is non-commercial. See: http://creativecommons.org/licenses/by-nc/3.0/. 


\section{Figures}
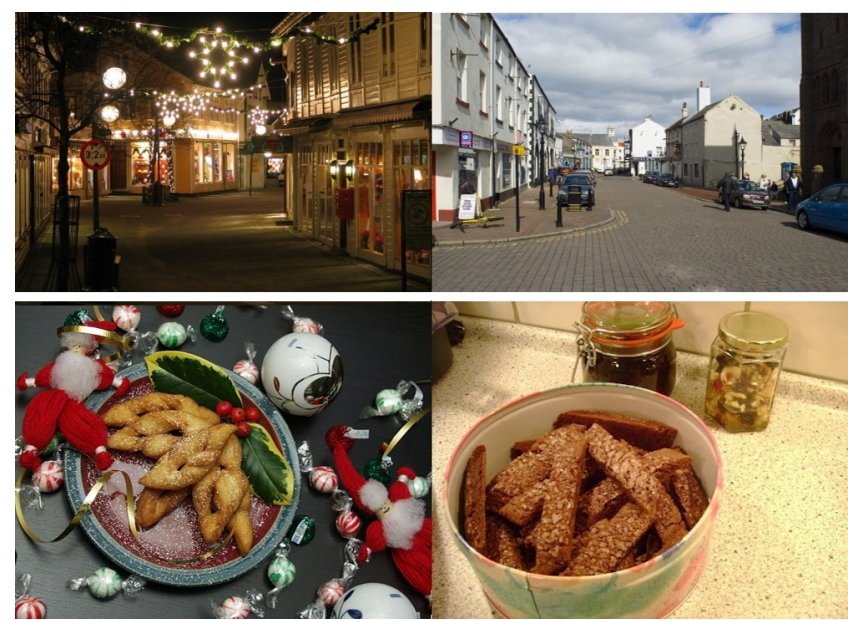

Fig 1 Four examples from image series viewed by participants, which represent images with and without Christmas theme
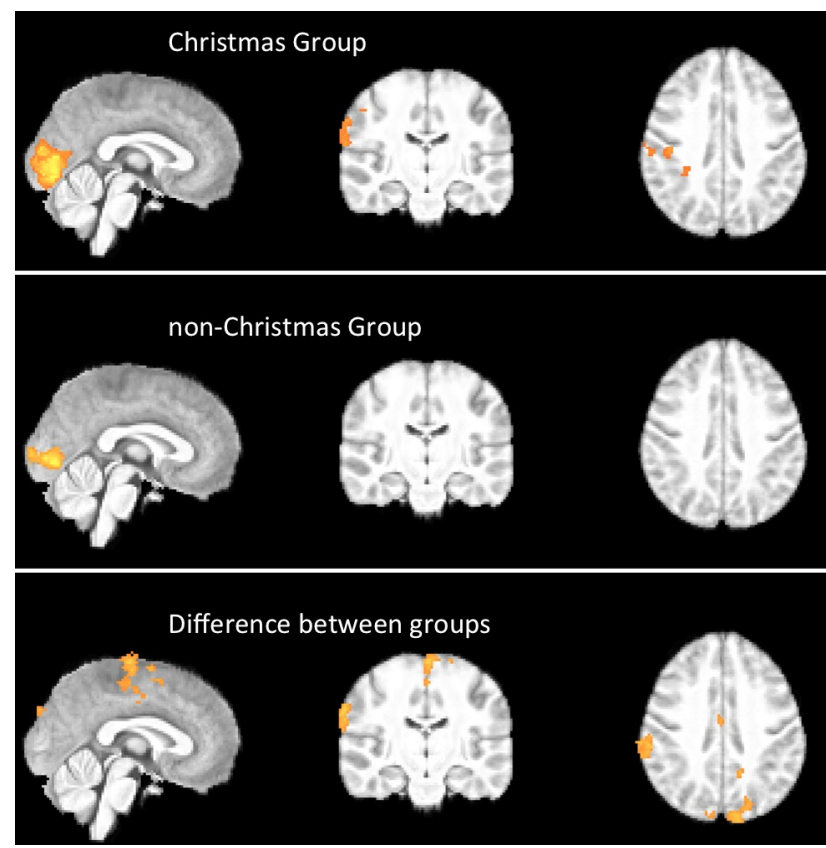

Fig 2 Activation maps showing areas of significant increases in cerebral activity while participants viewed images with a Christmas theme as opposed to everyday images. Results presented are of a group analysis of participants from the "Christmas group" (top row) and the "non-Christmas group" (middle row). Results of an analysis between groups (bottom row) show that the Christmas group had significantly higher activation (increase in cerebral activity) when viewing Christmas images. Results are shown according to radiological convention-that is, the right side on the coronal and transverse sections represent the left side of the patients and vice versa 


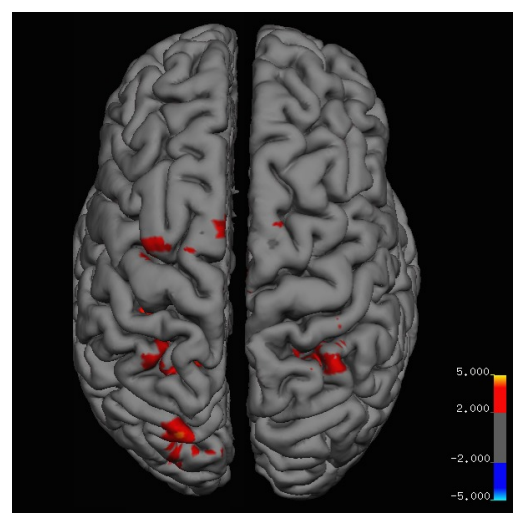

Fig 3 Cerebral areas where the "Christmas group" had a significantly higher increase in cerebral activity than the "non-Christmas group" while images viewed had a Christmas theme. The color scale is of z values representing response of "Christmas group" relative to "non-Christmas" as a control group. In contrast, there were no significant clusters where the "non-Christmas" group had a significantly higher activation than the "Christmas group" in response to Christmas images. 